Mots. Les langages du politique

$116 \mid 2018$

Dire ou ne pas dire la « race » en France aujourd'hui

\title{
Mariage pour tous dans la presse : itinéraire d'une nomination (2012-2013)
}

"Mariage pour tous" in the French press: the story of a naming process

(2012-2013)

«Mariage pour tous» en la prensa francesa: itinerario de una nominación

(2012-2013)

Martin Barrangou

\section{OpenEdition}

Journals

Édition électronique

URL : https://journals.openedition.org/mots/23066

DOI : $10.4000 /$ mots. 23066

ISSN : 1960-6001

Éditeur

ENS Éditions

Édition imprimée

Date de publication : 20 mars 2018

Pagination : 75-90

ISSN : 0243-6450

Référence électronique

Martin Barrangou, «Mariage pour tous dans la presse : itinéraire d'une nomination (2012-2013) », Mots. Les langages du politique [En ligne], 116 | 2018, mis en ligne le 23 février 2020, consulté le 22 avril 2022. URL : http://journals.openedition.org/mots/23066 ; DOI : https://doi.org/10.4000/mots.23066 


\section{Mariage pour tous dans la presse : itinéraire d'une nomination (2012-2013)*}

Dans une société démocratique traversée par des discours en tous genres, la défense de nouveaux droits peut se traduire par la naissance de nouvelles expressions. Les médias peuvent rester indifférents à ces créations ou les projeter à travers «un processus de mise en forme, de mise en scène et de mise en sens» (Neveu, Quéré, 1996, p.10). Des enjeux politiques et sociaux soustendent ces débats autour des mots, ces discussions métalinguistiques qui mettent au jour l'importance de l'acte de nomination des événements et de ses acteurs (Koren, 2016).

Notre étude, située dans le cadre de l'analyse du discours praxématique, théorie de la praxis linguistique et de la production du sens en discours, analyse le fonctionnement argumentatif de la nomination mariage pour tous dans la presse et dans le cadre du moment discursif (Moirand, 2007) établi à partir de l'annonce de l'engagement no 31 de François Hollande (janvier 2012) jusqu'à l'adoption de la loi à l'Assemblée nationale (avril 2013). Les limites temporelles proposées ne constituent pas des moments statiques où le sujet tendrait à s'épuiser. Nous sommes conscient de l'évolution des objets de discours au-delà du moment discursif.

Le corpus analysé se compose de types textuels divers : articles, chroniques, lettres de lecteurs, tribunes, etc., publiés par cinq quotidiens nationaux français disponibles en ligne et choisis en raison de leur positionnement républicain : Le Figaro (droite), Libération et L'Humanité (gauche), Le Monde (centre gauche) et La Croix (catholique).

Cet article analyse les enjeux du surgissement dans la presse de la nomination mariage pour tous et s'articule en quatre temps : dans un premier moment, nous retracerons l'évolution de l'institution matrimoniale ainsi que

* Je tiens à exprimer mes plus sincères remerciements aux relecteurs anonymes qui ont donné de leur temps précieux et de leur expertise contribuant, grâce à leurs remarques, à améliorer la qualité de cet article.

Instituto de Enseñanza Superior en Lenguas Vivas « Juan R. Fernández» martinbarrangou@gmail.com 
les redéfinitions du praxème mariage à travers les siècles. Nous essaierons de montrer ensuite que les désignations mariage homosexuel, mariage gay, mariage homo, etc., qui côtoient dans la presse la nomination mariage pour tous, témoignent d'une opposition entre altérité et égalité. Nous suivrons le parcours de la nomination mariage pour tous depuis sa genèse jusqu'à son installation en discours, et nous aborderons finalement les mécanismes d'appropriation de cette nomination par les locuteurs, ainsi que sa remise en question, car cette nomination est située au carrefour de forces contraires : tendance au figement et point de départ de la créativité langagière à travers des défigements, instances de production discursive source de divers positionnements.

Un bref rappel du contexte dans lequel la nomination mariage pour tous est mise en circulation permettra de situer notre étude.

\section{Le mariage des couples de même sexe : du projet à la loi}

Le mariage des couples de même sexe n'est pas en 2012 un sujet nouveau pour la société française. Pourtant, à l'exception de quelques revendications marginales pendant les années 1970-1980, la contre-culture du militantisme homosexuel considérait le mariage traditionnel comme une institution bourgeoise (Carnac, 2015). Ce n'est que depuis les années 1990 et 2000 que se dessinent les premières revendications en ce sens de la part de plusieurs associations (Paternotte, 2011).

Ces revendications, que l'avènement du PACS projette dans l'espace public en 1999, ouvrent le débat politique et religieux (Carnac, 2015). Des propositions de loi se succèdent pendant les années qui suivent (Portier, Béraud, 2015), jusqu'à ce que le 26 janvier 2012, lors de la campagne électorale, François Hollande présente l'engagement n 31 de son projet : "J'ouvrirai le droit au mariage et à l'adoption aux couples homosexuels». Le contexte discursif proclame l'égalité : "l'âme de la France, c'est l'égalité »; "Je veux lutter sans concession contre toutes les discriminations et ouvrir de nouveaux droits» (F. Hollande, programme officiel, 2012). C'est alors que la «controverse sociopolitique», c'est-à-dire «un conflit durable relatif à une décision publique et se dispersant dans une pluralité d'arènes académiques, gouvernementales, parlementaires, judiciaires, militantes, etc. »(Rennes, 2011, p. 38) voit le jour.

Après la victoire de F. Hollande en mai 2012, le projet de mariage des couples de même sexe connaît un tournant. La ministre de la Justice, Christiane Taubira, l'annonce le 10 septembre dans le quotidien La Croix, soulevant des objections de la part de certains à droite, qui réclament un référendum, et de la part des autorités catholiques, très présentes dans le débat (Carnac, 2015).

Après une première mobilisation locale au mois d'octobre, une manifestation nationale, prélude à la naissance du collectif «La Manif pour tous», rallie 
les opposants le 17 novembre (Portier, Béraud, 2015). À la suite des auditions du mois d'octobre à l'Assemblée nationale, le projet est en effet passé en Conseil des ministres le 7 novembre. La confrontation s'aggrave au mois de janvier et de février où détracteurs et défenseurs alternent les mobilisations. Le 2 février, l'article 1 est voté, et le 23 avril, le Parlement adopte le projet de loi. La France devient ainsi le neuvième pays européen à autoriser le mariage de personnes de même sexe.

\section{Mariage : le mot et l'institution}

Les cinq quotidiens analysés ici relaient la controverse : les désignations mariage homosexuel, mariage gay, mariage homo, etc. y côtoient une nouvelle nomination, mariage pourtous, dont les actualisations de sens témoignent de l'existence d'un objet de discours (Sitri, 2003) particulier et conflictuel.

Pour expliquer ce conflit autour des façons de nommer le mariage des couples de même sexe, nous ne saurions chercher du côté d'une linguistique considérant les mots pourvus d'un sens déjà fixé en langue. Nous considérons que «la seule voie scientifiquement valide et épistémologiquement acceptable est l'observation des comportements linguistiques effectifs » (Apothéloz, Reichler-Béguelin, 1995, p. 231) liés à des conditions de production définies à travers l'échange que les pratiques sociales instaurent entre le locuteur et son environnement (Siblot, 2001, p. 196-197). La nomination relève de l'actualisation discursive et constitue un geste à « dimension performative », permettant la catégorisation d'un référent dans une classe identifiée du lexique ou l'innovation néologique (Détrie, Siblot, Vérine, 2001). C'est cette distinction au niveau de la production qui permet de séparer l'espace correspondant à la dénomination comme « usage à un moment donné qui s’impose parmi des nominations » de celui de la nomination qui «permet de prendre en considération des phénomènes discursifs et sémiotiques sous-jacents à l'appréhension même des signes par les sujets parlants» (Longhi, 2015, p.5).

En effet, la loi d'ouverture du mariage pour les couples de personnes de même sexe implique une redéfinition de cette institution. L'enjeu est important : dans la controverse dont l'identité discursive est marquée par « un nœud d'arguments contradictoires récurrents qui [traversent les] différentes arènes du débat public » (Rennes, 2007, p. 91), le conflit entre défenseurs et détracteurs de la réforme passe par un acte symbolique d'appropriation à travers la définition d'un objet de discours fuyant. Ainsi, «l'acte de nomination pose un rapport au référent [ce qui le rend] susceptible d'orienter le jugement qu'adoptera l'allocutaire par rapport à ce référent » (Détrie, Siblot, Vérine, 2001, p. 37) et met face à face les mécanismes de persuasion de chaque locuteur. Quels sens accorder alors à l'objet de discours nommé le mariage? 
La description définie le mariage ne renvoie pas à la même réalité selon les locuteurs qui la prennent en charge. Pour les opposants à la réforme, le nom porterait un sens immanent et stable à travers le temps. Certains s'interrogent ainsi sur «le sens du mariage » et insistent sur le fait qu' «il est indispensable de rappeler la signification du mariage, anthropologiquement, juridiquement et socialement» (La Croix, 01/07/12).

À l'étranger, dans des pays où une réforme similaire se prépare, on défend cette signification prétendument unique, «vraie» :

(1) [...] l'archevêque Vincent Nichols, chef de l'église catholique en Angleterre et au Pays de Galles, et l'archevêque du district de Southwark à Londres, Peter Smith, demandent aux catholiques «de faire tout ce qu'ils peuvent pour s'assurer que la vraie signification du mariage ne soit pas perdue pour les générations futures». (La Croix, 01/07/12, nous soulignons)

Cette définition immuable garantirait l'existence d'un référent immuable aussi. Que la modification de cette réalité advienne sans conséquences ne peut se concevoir qu'au prix d'une «illusion». Une redéfinition impliquerait donc une rupture du sens instituant des réalités distinctes, un «nouveau type » issu d'un original :

(2) [...] l'archevêque de Paris [Mgr Vingt-Trois] a dit notamment avoir attiré l'attention de la garde des Sceaux sur «l'illusion» consistant à «affirmer que l'on puisse ouvrir le mariage sans le transformer. L'ouverture du mariage à des personnes homosexuelles va entraîner une transformation assez large des définitions de l'état civil et du Code civil. Ce sera donc un nouveau type de mariage ». (Le Figaro, 19/09/2012, nous soulignons)

Cette «transformation» risque de "dénaturer le sens du mariage» ( $L e$ Figaro, 28/12/2012) selon un courrier de l'Association des parents d'élèves de l'enseignement libre (APEL) aux présidents académiques et régionaux et se traduit par l'amenuisement de cette «nature » et la scission de l'institution :

(3) Selon eux [l'archevêque Vincent Nichols et l'archevêque Peter Smith], légaliser les unions homosexuelles changerait sa "nature même» en le réduisant "à un engagement entre deux personnes». (Libération, 12/03/2012, nous soulignons)

(4) Hétéros et homos sous le même toit, qu'est-ce que l'institution du mariage si elle est partagée en deux? Une institution qui ne fera plus l'objet d'un monopole mais d'un partage de biens! (Le Figaro, 13/01/2012, nous soulignons)

Le mariage est défini par des concepts abstraits, transcendants : à en croire les exemples suivants, le mariage serait un «acte fondateur» (7), porteur des «repères» (6) et des «fondements» (7) de la société, une «valeur fondamentale » (5). La réforme constituerait « une menace», voire « une véritable agression» $(6)$ : 
(5) Pour la droite, le mariage est une valeur fondamentale, ciment de la société. «Une institution», "un symbole social», a répété le garde des Sceaux [Michel Mercier], en juin 2011, lors de l'examen à l'Assemblée de la proposition de loi PS sur l'ouverture du mariage civil aux couples de même sexe. (Libération, 10/5/2012, nous soulignons)

(6) Face à la crise et faute de destin collectif, beaucoup de gens s'accrochent $a u$ mariage, vécu comme un élément d'identité, et à la famille, vécue comme une cellule protectrice, répond Bruno Le Maire. Ils ressentent le mariage des homosexuels comme une menace sur ces derniers repères. La crispation est encore plus forte chez les catholiques, qui y voient une véritable agression contre leurs valeurs. (Le Monde, 10/01/2013, nous soulignons)

(7) Brigitte Kuster, maire UMP du XVIIe arrondissement de Paris, a dénoncé «un débat escamoté » et demandé «l'abrogation du texte [...]. On touche au fondement de la société dont l'acte fondateur est le mariage. Que deux personnes de même sexe veuillent s'unir, c'est normal, mais le mariage, c'est dans le but de créer une famille et on voit bien là la difficulté», a ajouté Mme Kuster. (La Croix, 15/12/12, nous soulignons)

L'essentialisme de la définition soustrait symboliquement la nomination à la tension lexico-idéologique, afin de préserver un nom au référent aussi immuable que lui, à moins de parler d'un autre mariage. Comme l'archevêque de York, qui déclare :

(8) Je ne pense pas que ce soit le rôle de l'État de définir ce qu'est le mariage. Il est situé dans la tradition et l'histoire, et vous ne pouvez pas [le changer] du jour au lendemain, peu importe comment vous êtes puissants [sic]. (Le Monde, 12/06/2012)

Ainsi, la définition du mariage impliquerait une « institution sociale essentielle» (La Croix, 09/02/12) à l'abri des changements.

Cette conception essentialiste et idéale des institutions de la société que la langue décrit considère le mariage comme un symbole «intangible et sacré » (Libération, 20/09/2012), une essence que la langue ne ferait qu'étiqueter. Cependant, le rapport à travers le temps entre les pratiques sociales et le lexique est tout autre, car celui-ci fournit «un ensemble de dispositifs extrêmement malléables, continuellement travaillés dans et par les discours » (Apothéloz, Reichler-Béguelin, 1995, p. 235).

Le lexique en diachronie rend compte de l'évolution des pratiques sociales et de leur redéfinition permanente. Dans cette adéquation référentielle, a lieu surtout « une contrainte socio-culturelle davantage qu'ontologique » (Apothéloz, Reichler-Béguelin, 1995, p. 236). En effet, les connaissances issues de la rencontre des réalités et des praxis enregistrées comme des programmes de sens constituent le sémantisme des catégories nominales qu'il s'agisse d'objets ou de pratiques sociales (Siblot, 1997). 
Le verbe marier, du latin impérial maritare, provient du lexique de l'agriculture et signifie d'abord "unir des arbres à la vigne». Il est dérivé de maritus, adjectif dont le sens passe de «accouplé, uni », dans le domaine agricole, à «conjugal, nuptial», dans la langue poétique. En tant que nom, le terme remplace vir qui désigne l' "homme-époux» (Dictionnaire historique de la langue française, 2000, p. 2139). Le mot mariage (déverbal de marier) revêt au XIIe siècle un sens religieux, celui d'un sacrement indissoluble et transcendant différent d'autres types d'union. Jusqu'à la Renaissance, les «mariages arrangés » et « de raison » se différenciaient, du point de vue juridique, du « mariage d'amour », les décisions de la famille prévalant sur le libre choix des individus et leurs sentiments dans le couple. Mais cette conception du mariage cède (sans disparaître pour autant) devant celle d'une «alliance entre deux personnes».

Au XVIIIe siècle, la Constitution de 1791, dans son article 7, fait du mariage « un contrat civil». Son ouverture et sa sécularisation bénéficient aux citoyens autres que les catholiques et impliquent une redéfinition face à la tradition. Jean Étienne Marie Portalis, rédacteur du Code Napoléon de 1804, repense ainsi les rapports entre le mariage et la cellule familiale : c'est «la conception moderne de la parenté et du droit civil de la famille» (Théry, 2011). Cependant, les liens unissant le mariage à la filiation évoluent et au xx $x^{e}$ siècle la filiation s'autonomise par rapport au mariage (fin de l'interdiction de recherche en paternité pour les enfants nés hors mariage, fin de la différence entre filiation légitime et naturelle).

Ainsi, l'institution du mariage est sans cesse redéfinie tout au long de son histoire en fonction de ses nouveaux rapports à la laïcité, à la famille et au couple :

Le grand enjeu du débat actuel sur le mariage pour tous et l'homoparentalité, c'est qu'il s'agit d'une métamorphose institutionnelle sans précédent [...]. En instituant le mariage de même sexe, il ne s'agit pas de faire accéder au mariage une minorité qui en était exclue mais bien de redéfinir pour tous l'institution même du mariage. (Portier, Théry, 2015)

Le conflit naît donc de l'appropriation par différents acteurs d'une même nomination dont le sens est soumis à la recherche d'un consensus difficile à atteindre. C'est dans l'énonciation en tant qu'acte que réside la construction du sens permettant au sujet de mettre en avant «la responsabilité énonciative active du locuteur, que son choix se porte sur une dénomination lexicalisée ou sur une désignation discursive de son cru» (Koren, 2016, p.4).

Face aux désaccords que suscite le nom mariage, un espace dénominatif béant accueille, pendant la période analysée, différentes désignations conflictuelles que la presse fait circuler. 


\section{Altérité versus égalité}

Deux types de désignations opposent dans l'arène médiatique une conception spécifiante à une conception universaliste du mariage. D'une part, les désignations qui correspondent au praxème mariage suivi d'un prédicat de nomination appartenant au fondement praxique de la sexualité (Siblot, 1998) et actualisé par des praxèmes tels que homosexuel, gay, homo, lesbien (présentant le mariage comme un mariage autre) et, d'autre part, les désignations appartenant à un contexte discursif où le trait sémantique «égalité » est actualisé par le syntagme prépositionnel pour tous, caractérisant indirect ${ }^{1} \mathrm{du}$ praxème mariage.

Employer un prédicat de nomination dont la valeur praxique spécifie le nom mariage inscrit dans le discours la présence de l'altérité. Il en va ainsi de mariage homosexuel, mariage homo, mariage gay ou mariage lesbien. Certains locuteurs introduisent à travers ces désignations - parmi lesquelles mariage homosexuel apparaît comme prototypique dans les cinq quotidiens analysés - la notion d'un mariage à part. C'est notamment le cas d'un article dans lequel le cardinal André Vingt-Trois avertit de la création d'un «nouveau type de mariage » (La Croix, 18/09/12).

Pour certains détracteurs, il ne s'agit pas d'une ouverture de l'institution déjà existante, mais de la création d'une nouvelle réalité :

(9) L'ex-ministre Christine Boutin, présidente du Parti chrétien-démocrate, a estimé également que l'institution d'un mariage homosexuel serait irréversible. ( $\mathrm{La}$ Croix, 05/11/12, nous soulignons)

En témoigne l'emploi de l'article indéfini, «qui a un sens réel "indéterminé", dans la mesure où tout en actualisant le substantifil ne l'identifie pas » (Kleiber, 1983, p. 87).

Ainsi, pour les opposants, il s'agit d'un "projet de nouveau mariage» (Le Figaro, 24/10/2012, nous soulignons). Ce genre d'argument présente le mariage «sous une forme cristallisée, invariable aussi bien dans l'histoire que dans la géographie humaine des institutions sociales» (Cervulle, 2013, p. 209).

Le conflit est repérable dans les discours lorsque les défenseurs de la réforme récusent l'altérité pointée par les désignations mariage homosexuel, homo ou gay:

(10) "Cela confirme la philosophie du texte qui est d'ouvrir le mariage aux homosexuels et non pas de créer un mariage homosexuel», a expliqué le rapporteur du projet, Erwann Binet. (La Croix, 17/01/13)

1. Nous suivons la terminologie de Marc Wilmet. 
Ainsi, ces expressions, liées à l'altérité, à «une sexualité minorisée» (Chetcuti-Osorovitz, Girard, 2015) désignent un mariage autre inscrit dans le caractérisant spécificateur ${ }^{2}$. Elles l'inscrivent en outre dans une opposition plus générale entre le même et l'autre, comme semble le confirmer la présence de certains sujets de société réputés sensibles dans les argumentaires démontrant la mise en danger des « valeurs traditionnelles » de la société. Ainsi, dans une dépêche de Libération, Nicolas Dupont-Aignan qualifie de «concours Lépine de petites propositions minables» des sujets tels que «le halal, le mariage homosexuel, ou l'euthanasie » (Libération, 29/03/2012). L'ouverture du mariage désignée comme mariage homosexuel ou mariage gay constituerait de ce fait l'un des chainons de cette «série de promesses [...] potentiellement destructrices» (Libération, 13/05/2012). Un «mariage de cette nature» est pour certains, selon l'argument de la pente glissante, une «apocalypse» (Le Figaro, 21/12/2012), une "boîte de Pandore» (Le Figaro, 13/12/2012) ou un «cheval de Troie» (Le Monde, 26/10/2012).

Pendant les premiers mois du conflit, l'expression $X$ pourtous apparaît dans des gloses effaçant au contraire cette notion d'altérité. Il y a là une formule au sens d'Alice Krieg-Planque, qui joue un rôle structurant des événements qu'elle désigne en discours 3 . La notion de formule permet de mettre en lumière les étapes de la formation de la nomination mariage pour tous, porteuse d' " un caractère [variablement] figé; une existence en discours; une valeur de référent social; une dimension polémique» (Krieg-Planque, 2012, p.111).

(11) Ce n'est pas le mariage gay, c'est le mariage tout court. Cela va se traduire simplement par une loi qui lèvera toute ambiguïté sur le fait qu'un couple est composé de deux personnes, quel que soit leur sexe. Le même droit pour tous. (Libération, 03/03/2012, nous soulignons)

(12) la future loi ne créera pas un autre mariage, qui serait homo, elle ouvrira simplement le mariage - le même pour tous, indivisible - aux couples de même sexe. (Libération, 18/07/2012, nous soulignons)

Inscrite dans le temps du discours, une formule est souvent devancée par des formes qui annoncent sa survenue. Ainsi, le syntagme prépositionnel pour tous qui glose le nom mariage dans une opération de fixation du sens précède l'émergence de la nomination mariage pour tous qui s'oppose à l'altérisation des autres nominations :

2. Michel Foucault situe au XIXe siècle la naissance du terme homosexuel en tant que catégorie de la scientia sexualis et il le décrit comme faisant partie d'une «spécification nouvelle des individus », faisant de l’homosexuel « une espèce» (Foucault, 1976, p. 59).

3. Alice Krieg-Planque la définit ainsi : «[...] une séquence verbale, formellement repérable et relativement stable du point de vue de la description linguistique qu'on peut en faire, se met à fonctionner dans les discours produits dans l'espace public comme une séquence conjointement partagée et problématique. Portée par des usages qui l'investissent d'enjeux socio-politiques parfois contradictoires, cette séquence connaît alors un régime discursif qui fait d'elle une formule [...]» (Krieg-Planque, 2003, p.14). 
(13) La jeune femme a envie de se marier. Et corrige lorsqu'on parle de «mariage gay»: "C'est mariage pour tous. On ne veut pas un truc spécial, on veut la même chose que les autres.» "É-ga-li-té des droits», reprend-elle, en détachant chaque syllabe pour marquer sa détermination. (Le Monde, 10/02/2012)

Si mariage pour tous tend à se stabiliser à partir de septembre 2012, son comportement syntaxique et sémantique peut être décrit en deux moments : sa genèse, où la formule est annoncée par les syntagmes prépositionnels pour tous (les couples), à tous (les couples), de tous (les couples), puis son installation dans les discours avec un degré de figement croissant, ce qui suscite de nouveaux désaccords à mesure que la publicisation la transforme en un nouvel enjeu du débat.

\section{Mariage pour tous : genèse d'une nomination}

Pendant la campagne présidentielle de 2012, dans certains discours politiques, les revendications s'expriment par les syntagmes le droit au mariage et l'ouverture du mariage :

(14) Parité et libertés : François Hollande a rappelé l'engagement socialiste du "droit au mariage et à l'adoption pour tous les couples» sans question de sexe [...]. (Le Monde, 22/01/2012, nous soulignons)

Ce sont surtout les quotidiens Libération, Le Monde et L'Humanité qui, dès les premiers mois de notre moment discursif, font circuler les syntagmes prépositionnels pour tous les couples, à tous les couples, de tous les couples dans la dépendance des constructions droit au mariage, accès au mariage, ouverture du mariage :

(15) Dans la perspective des élections à venir, le Front de gauche s'engage à ce que le droit au mariage pour tous les couples, ainsi que la reconnaissance légale des familles homoparentales dans le cadre du droit commun de la filiation, soient reconnus en France [...]. (L'Humanité, 10/02/2012, nous soulignons)

(16) En novembre, six jeunes secrétaires généraux de l'UMP appelaient à l'ouverture du mariage à tous les couples dans une tribune publiée par L'Express. (Le Monde, 24/01/2012, nous soulignons)

Quels sens véhiculent donc les syntagmes prépositionnels pour tous les couples et à tous les couples? L'adjectif indéfini tous désigne «l'ensemble, la totalité de, sans excepter une unité, le plus grand nombre de» (Le Petit Robert, 2012). Cependant, l'emploi de cet indéfini, voué à transmettre la notion d'égalité parmi les citoyens, ne permet pas de cerner, surtout dans ce premier moment de genèse, l'identité des récipiendaires de ces nouveaux droits. L'ambiguïté est donc mise en évidence par des gloses de fixation du sens dont le 
but, pour l'énonciateur, est de «protéger son dire» de cette pluralité potentielle des sens qui habite les mots et qui peut faire surface dans le discours à tout moment (Authier-Revuz, 1994):

(17) Nous demandons l'accès au mariage civil pour tous, y compris pour les couples de même sexe. C'est-à-dire l'égalité des droits. (Libération, 13/01/2012, nous soulignons)

(18) Engagée dans un combat pour l'égalité, la FSU demande depuis longtemps l'ouverture du mariage et de l'adoption à tous les couples, quelle que soit leur orientation sexuelle. (L'Humanité, 22/02/2012)

(19) Il s'agit avant tout de lutter contre les discriminations. Marie-George Buffet a ainsi présenté en 2005 la première proposition de loi ouvrant le mariage à tous sans distinction de sexe. (Le Figaro, 13/01/2012)

Ces gloses orientent l'interprétation des syntagmes : il s'agit d'ouvrir le mariage aux couples de même sexe exclus d'un tel droit. L'effacement des gloses vers le mois de mai 2012 cède la place aux syntagmes pour tous et à tous. Il s'établit ainsi au sein du paradigme une opposition entre les nominations qui se construisent à partir du nom mariage + syntagme adjectival (homosexuel, homo, gay, etc.) et mariage + syntagme prépositionnel (pour tous les couples, à tous les couples). Ces structures syntagmatiques actualisent en discours un sens particularisant opposé à un sens inclusif porteur d'ambiguïté aux yeux de certains locuteurs. Car l'emploi du pronom tous, non marqué en genre, nourrit l'argument qui dénonce une atteinte à la « différence des sexes » ayant traditionnellement caractérisé le mariage. En outre, l'indéfini s'ouvre à des sens multiples que ceux qui récusent la nomination mariage pour tous, aussi bien du côté des opposants que des défenseurs du projet de loi, signalent :

(20) Et voilà que le «mariage homosexuel» se présente aujourd’hui sous le label de «mariage pour tous». Outre la banalité de l'expression, proche du slogan publicitaire ou de la banderole de manifestation - et indépendamment de l'objection de fond que l'on peut avoir face à ce qu'elle signifie -, il faut en tout cas remarquer qu'elle est inexacte car, fort heureusement, cette nouvelle union élargie ne s'ouvre pas à tous. Il reste des interdits (liés à l'âge, aux liens de parenté...). (La Croix, 11/09/12)

Les hésitations témoignant d'une prise de distance atteignent aussi cette formule. Les rectifications, les accumulations mais aussi l'emploi des guillemets sont la preuve d'un tel positionnement :

(21) Alors que le gouvernement Ayrault, après quelques cafouillages de calendrier, entend présenter son projet de loi sur le mariage pour tous (également appelé mariage "gay», "entre personnes du même sexe», "universel») fin octobre en Conseil des ministres, les sénateurs verts dégainent. (Libération, 09/09/2012, nous soulignons) 
(22) Trop de mes confrères, à mon goût, ont en effet interprété la sortie de la ministre [...] comme une piquante réponse à la croisade organisée par leurs éminences contre le mariage homo (ou "gay», ou "pour tous»). (Libération, $6 / 12 / 2012$, nous soulignons)

(23) Ils auront tout essayé pour empuantir l'atmosphère. Le 18 novembre dernier, les anti-mariage pour tous (ou mariage homo, comme ils disent) ont de nouveau remué la poussière. (L'Humanité, 05/12/2012, nous soulignons)

Ces guillemets se multiplient dès septembre dans les cinq quotidiens analysés, signalant une mise à distance des locuteurs qui ne prennent pas en charge l'expression, ce qui témoigne de l'installation de la formule mariage pour tous comme nomination à part entière, opposée dans l'arène discursive aux autres façons de nommer le mariage des personnes de même sexe. En effet, dans notre corpus, les guillemets entourant le syntagme prépositionnel pour tous cèdent la place au-delà du mois de décembre 2012 aux guillemets entourant le syntagme nominal complet, le mariage pourtous, comme un nouvel objet discursif.

(24) L'Association des maires ruraux de France (AMRF) s'est déclarée hostile vendredi à l'instauration d'un «droit de conscience» permettant aux maires de ne pas célébrer de mariages homosexuels si la loi sur le «mariage pour tous» était adoptée. (Libération, 26/10/2012)

Certes, cette nomination suscite de nouvelles controverses, repérables au niveau métadiscursif. Ainsi, l'emploi autonymique de mariage pour tous interroge les origines de cette expression :

(25) «Au fait, d'où vient cette expression “mariage pour tous” ?» La question suscite une moue sceptique chez un membre du cabinet de Christiane Taubira et des regards perplexes parmi le staff de Jean-Marc Ayrault. (Le Figaro, 26/09/2012)

L'emploi autonymique opère l'inscription de la nomination dans le débat à travers une mise en relief :

Dans tout fait de réflexivité autonymique, il y a un signe qui s'impose comme objet, propulsé sur le devant de la scène comme «personnage» auquel le dire fait référence, sortant par là de son rôle de rouage ordinaire de la machinerie du dire, voué à l'effacement dans l'accomplissement de sa fonction ordinaire de médiation (Authier-Revuz, 2011, p.71).

Cet indice de «saillance politico-médiatique » surgit d'un «travail conjoint des acteurs politiques et des journalistes», par lequel «le mot vient occuper le devant de la scène dans la mesure où il est érigé en objet du désaccord : il devient pour une durée médiatique limitée ce à propos de quoi il y a lieu de s'affronter» (Micheli, 2013). Les différentes tentatives de classement de la nomination mariage pour tous rendent compte de sa dimension argumentative, car 
elle est analysée en tant qu'objet discursif dans « un travail de cadrage médiatique» réalisé par les journalistes :

(26) Que vous inspire le terme de «mariage pour tous»? [journaliste]

Cette expression ne veut pas dire grand-chose. [...] Le «mariage pour tous» fait partie de ce vocabulaire qu'aime inventer la gauche. Il y a, dans la majorité actuelle, beaucoup de termes fétiches de ce type [Axel Poniatowski]. (Le Figaro, 26/12/2012)

Présentée en mention, la nomination, censée «inspirer», faire réagir, se constitue en enjeu que les locuteurs - défenseurs ou détracteurs de la réforme - analysent et critiquent en tant que "slogan absurde » (Le Figaro, 15/11/2012), «formule caricaturale» (Le Figaro, 19/02/2013) ou «formule [...] cache-sexe pour éviter l'expression de mariage gay » (Libération, 12/01/2013). Dans la polyphonie du discours, la présence des guillemets signale l'inadéquation que l'énonciateur éprouve par rapport à l'expression issue d'un discours qu'il ne reconnaît pas comme propre4. Ces guillemets sont accompagnés par une «modalité énonciative de redoublement du dire » (Authier-Revuz, 1994, p. 91). Ainsi, parmi les défenseurs du projet de loi, l'expression mariage pour tous peut être mise à distance. Pierre Galand l'explique dans sa tribune :

(27) Le débat qui agite votre landerneau à propos de ce que vous appelez pudiquement le «mariage pour tous» nous laisse pantois. (Libération, 13/01/2013, nous soulignons)

Mais il peut s'agir aussi de mettre en relief la rupture du rapport entre le mot et la chose :

(28) L'expression «mariage pour tous» est impropre. (Le Monde, 29/01/2013, nous soulignons)

\section{Défigement et productivité de la nomination}

En discours, la formule mariage pour tous ne saurait rester immuable dans ce contexte tendu. Lorsque les locuteurs s'en emparent, le défigement de la formule témoigne d'une validation ou bien d'une remise en question, toutes deux porteuses d'une valeur argumentative.

C'est le cas, lors des premières manifestations, du collectif «La Manif pour tous », qui réunit des opposants au projet de loi et dont le nom naît du détournement de l'expression de base pour interpeller aussi bien les défenseurs que les contempteurs du projet (Fracchiola, 2015).

4. Selon Jacqueline Authier, «l'élément autonyme constitue, dans l'énoncé où il figure, un corps étranger, un objet “montré” au récepteur; en ce sens qu’on peut considérer ces mots guillemetés comme "tenus à distance", en un premier sens, comme on tient à bout de bras un objet que l'on regarde et que l'on montre» (Authier-Revuz, 1981, p.127). 
Employée par les adversaires de la réforme, la valeur de revendication de la nomination mariage pour tous produit un effet d'écho lorsque les opposants exigent un «débat pour tous» (Le Figaro, 19/11/2012), se rassemblent dans un «camping pour tous» (Libération, 08/04/2013), ou réclament «l'emploi pour tous» (Le Monde, 21/12/2012). Cette même stratégie de revendication est employée par ceux qui, favorables au projet, proposent des affiches sur le site Affichespourtous.fr (L'Humanité, 12/12/2012) ou par les organisateurs des "Jeux pour tous » (Le Monde, 28/11/2012) dans le cadre des Gay Games 2018.

L'activité néologique engendre la création de hashtags circulant sur Twitter et par le moyen desquels les usagers expriment leur positionnement dans le débat en se rassemblant autour des mots-clés \#campingpourtous, \#Joggingpourtous (Le Figaro, 15/04/2013). Les tensions dans ces espaces de publication de messages courts ont été analysées par Maxime Cervulle et Fred Pailler, qui s’interrogent sur la façon dont la «politique affective» véhiculée par ces hashtags «active et valorise des rapports de force entre des acteurs à la fois individuels et collectifs» (Cervulle, Pailler, 2014).

En dernier lieu, la créativité du défigement se manifeste dans les banderoles déployées lors des manifestations : "Le divorce pour tous ( Libération, 16/12/2012), «le droit pour tous d'avoir le choix» (Le Figaro, 16/12/2012), "Liberté, égalité, fécondité pour toutes et tous»(Le Figaro, 16/12/2012). Là encore, le sens de la structure imitée ou subvertie est censé interpeller, faire réagir, voire provoquer pendant ces moments intenses de confrontation et de revendication des droits.

Dans cette étude de la nomination mariage pour tous réalisée à partir de cinq quotidiens nationaux français, nous avons voulu montrer l'émergence et les évolutions d'un objet de discours traversé par des enjeux idéologicodiscursifs et reflétant une construction sociale conflictuelle au niveau du dire.

Dans cette perspective, la nomination en tant qu'acte de parole nous a permis de constater que le dialogisme sous-tend la signifiance lexicale, phénomène qui participe de l'intelligibilité que les événements acquièrent pour les sujets qui les catégorisent en les nommant. C'est précisément cette dialectique du dire, cette construction partagée de la réalité à travers l'acte de nommer qui fait en même temps ressurgir l'altérité sous les différents positionnements dont les locuteurs font preuve.

Ainsi, le discours de la presse s'est révélé un espace propice dans lequel nous avons pu repérer la présence d'un paradigme apportant une vision spécifiante du mariage par l'emploi d'un caractérisant relié à la praxis sexuelle : mariage homosexuel, mariage homo, mariage gay, etc. Par rapport à ce paradigme, la nomination mariage pour tous qui actualise dans son programme de sens le trait sémantique égalité suscite le conflit au sein du discours. L'avènement de cette nomination qui se produit au fil des mois et par le biais des gloses explicatives tend progressivement à se fixer, suivant, d'un côté, l'appropriation 
conflictuelle que la nomination connaît chez les locuteurs et les commentateurs et, d'un autre côté, sa circulation dans la presse pendant les périodes de recrudescence du conflit social. Car, si la nomination mariage pour tous est, pendant la période étudiée, loin de faire l'unanimité par rapport au sens qu'elle véhicule, elle n'en est pas moins employée par les cinq quotidiens analysés dans cet article.

Nous avons ainsi constaté que la nomination mariage pour tous connaît une diffusion qui la transforme en carrefour, point de contact où se produit le dialogue tendu entre les énonciations qui la prennent en charge ou qui la récusent, comme l'indiquent les défigements de la nomination qui en constituent l'écho mémoriel.

Le dynamisme de toute nomination fondée sur la négociation du consensus social fournit de nouveaux questionnements sur le devenir de la nomination mariage pour tous en discours mais aussi en langue. Au-delà des limites fixées par notre corpus, nous avons constaté que Le Petit Robert (2014) ainsi que des dictionnaires en ligne tels que L'internaute et Wiktionnaire retiennent cette locution issue d'un moment historique et social situé :

Mariage pour tous, locution. Expression française caractérisant la loi du gouvernement de François Hollande permettant aux couples homosexuels de s'unir par le mariage. (L'internaute, http://www.linternaute.com/dictionnaire/fr/definition/ mariage-pour-tous/, consulté le 19 mars 2017)

Mariage pour tous, locution nominale, masculin. (France) Appellation utilisée pour désigner un projet de loi ouvrant le mariage et donc l'adoption aux couples homosexuels. (Wiktionnaire, http://fr.wiktionary.org/wiki/mariage_pour_tous, consulté le 19 mars 2017)

Formes historiques, culturelles du mariage. [...] Mariage pour tous, pour tous les couples, de sexe différent ou de même sexe. Mariage homosexuel, gay. (Le Petit Robert, 2014)

Ainsi, la présence de cette nomination dans les discours tend à se prolonger au-delà du conflit social et discursif de la période analysée dans ce travail, comme ce fut le cas pour d'autres nominations conflictuelles (purification ethnique ou intermittents) accueillies aujourd'hui dans les dictionnaires. En effet, ceux-ci, en tant que «lieux de fabrication de l'imaginaire de la langue», n'échappent pas à la dialectique qui unit les divers discours sociaux à l'histoire (Branca-Rosoff, 1997, p.151) et gardent, en ce sens, le souvenir de ces événements dont le caractère conflictuel s'exprime à travers les mots. 


\section{Références}

Apothéloz Denis, Reichler-Beguelin Marie-José, 1995, «Construction de la référence et stratégies de désignation », Travaux neuchâtelois de linguistique, nº 23, p. 227-271.

AUthIER-ReVUz Jacqueline, 2011, «Le fait autonymique : langage, langue, discours. Quelques repères », dans Parler des mots. Le fait autonymique en discours, J. AuthierRevuz, M. Doury et S. Reboul-Touré éd., Paris, Presses Sorbonne Nouvelle, p. 67-96.

- 1994, "L'énonciateur glosateur de ses mots : explicitation et interprétation », Langue française, $\mathrm{n}^{0} 1$, p. 91-102.

- 1981, "Paroles tenues à distance», dans Matérialités discursives, B. Conein et M. Pêcheux éd., Lille, Presses universitaires de Lille, p. 127-142.

BRANCA-Rosoff Sonia, 1997, "Guerre de religion et guerre sainte dans les dictionnaires français », Mots. Les langages du politique, n ${ }^{50}$, p. 151-156.

CARNAC Romain, 2015, «Les autorités catholiques dans le débat français sur la reconnaissance légale des unions homosexuelles (1992-2013) », dans Genre et christianisme : plaidoyers pour une histoire croisée, M. Brejon de Lavergnée et M. Della Sudda éd., Paris, Beauchesne, p. 375-409

Cervulle Maxime, 2013, «Les controverses autour du “mariage pour tous" dans la presse nationale quotidienne : du différentialisme ethno-sexuel comme registre d'opposition », L'Homme et la société, no 189-190, p. 207-222.

Cervulle Maxime, Pailler Fred, 2014, «\#mariagepourtous : Twitter et la politique affective des hashtags", Revue française des sciences de l'information et de la communication, n 4, http://rfsic.revues.org/717 (consulté le 12 février 2017).

Chetcuti-Osorovitz Natacha, GIRARD Gabriel, 2015, "L'appropriation d'une sexualité minorisée », Revue ¿ Interrogations ?, n²1, http://www.revue-interrogations.org/Lappropriation-d-une-sexualite (consulté le 25 janvier 2017).

DÉTRIE Catherine, SIBLOT Paul, VERIn Bertrand, 2001, Termes et concepts pourl'analyse du discours. Une approche praxématique, Paris, Honoré Champion.

FRACCHIOLLA Béatrice, 2015, "Violence verbale dans le discours des mouvements antagonistes : le cas de "Mariage pour tous" et "Manif pour tous" ", Argumentation et analyse du discours, n¹4, http://aad.revues.org/1940 (consulté le 11 février 2017).

Fou Cault Michel, 1976, Histoire de la sexualité, I. La volonté de savoir, Paris, Gallimard.

KLEIBER Georges, 1983, «Article défini, théorie de la localisation et présupposition existentielle», Langue française, nº57, p. 87-105.

KOREN Roselyne, 2016, "Introduction », Argumentation et analyse du discours, n 17 , http://aad.revues.org/2295 (consulté le 29 décembre 2016).

KrIeg-Planque Alice, 2012, Analyser les discours institutionnels, Paris, Armand Colin.

- 2003, Purification ethnique, Paris, CNRS Éditions.

LoNGHI Julien, 2015, «Stabilité et instabilité dans la production du sens : la nomination en discours ", Langue française, $\mathrm{n}^{\circ} 188$, p. 5-13.

MICHELI Raphaël, 2013, "Les querelles de mots dans le discours politique : modèle d'analyse et étude de cas à partir d'une polémique sur le mot “rigueur" ", Argumentation et analyse du discours, nº10, http://aad.revues.org/1446 (consulté le 10 février 2017).

MoIRAND Sophie, 2007, Les discours de la presse quotidienne. Observer, analyser, comprendre, Paris, PUF.

NeVEu Érik, QuÉRÉ Louis, 1996, «Présentation », Réseaux, nº 75, http://enssibal.enssib. fr/autres-sites/reseaux-cnet/75/sommai75.html (consulté le 3 avril 2015). 
PATERnotTe David, 2011, Revendiquer le «mariage gay». Belgique, France, Espagne, Bruxelles, Éditions de l'université de Bruxelles.

Portier Philippe, BÉraud Céline, 2015, Métamorphoses catholiques. Paris, Éditions de la Maison des sciences de l'homme.

PORTIER Philippe, ThÉRY Irène, 2015, «Du mariage civil au “mariage pour tous". Sécularisation du droit et mobilisations catholiques », Sociologie, n ${ }^{1}$, vol. Vl, http://sociologie.revues.org/2528 (consulté le 10 mars 2016).

RENNES Juliette, 2011, "Illégitimer des distinctions en droit. Stratégies politiques et enjeux épistémologiques », Politix, nº 94, p. 35-57.

- 2007, «Analyser une controverse. De la science politique à l'étude argumentative», dans Analyse de discours et sciences humaines et sociales, S. Bonnafous et M. Temmar éd., Paris, Ophrys, p. 91-107.

SiBlot Paul, 1998, «De l'un à l'autre. Dialectique et dialogisme de la nomination identitaire », dans L'autre en discours, J. Brès, R. Delamotte-Legrand, S. Madray-Lesigne et al. éd, Montpellier, Université Paul Valéry, p. 27-43.

- 1997, «Nomination et production de sens : le praxème », Langages, n 127, p. 38-55.

SITRI Frédérique, 2003, L'objet du débat. La construction des objets de discours dans des situations argumentatives orales, Paris, Presses Sorbonne Nouvelle.

THÉRY Irène, 2011, «Mariage religieux et mariage civil : les christianismes et la laïcité », dans Sacrées familles!, M. Gross, S. Mathieu et F. Nizard éd, Paris, Érès.

WILMET Marc, 2003, Grammaire critique du français, Bruxelles, Duculot. 